\title{
Hairs and pores in low-Reynolds-number flows
}

\section{Nonlinear flow response of soft hair beds}

Authors: José Alvarado, Jean Comtet, Emmanuel de Langre and A. E. Hosoi Nature Physics 13, 1014-1019, (2017)

\section{Bending and Stretching of Soft Pores Enable Passive Control of Fluid Flows}

Authors: Jean-François Louf, Jan Knoblauch, and Kaare H. Jensen

Phys. Rev. Lett. 125, 098101 (2020)

\section{Recommended with a Commentary by Eleni Katifori, Department of Physics and Astronomy, University of Pennsylvania, Philadelphia PA}

From the subcellular cytoplasmic flows and to the circulation of blood and other biological fluids, most life is composed primarily of water which is typically in constant motion $[1,2]$. The management and control of that motion is paramount for the fitness of every organism. Many of the flows inside living organisms happen at low Reynolds numbers, and as such they are spared from the capriciousness of turbulence at high Re and other non-linearities at intermediate Re. However, that does not mean that the behavior of these low Reynolds number flows is always ordinary. The papers by Alvarado et al. and Louf et al. presented in this commentary explore two instances of the rich phenomenology of these flows. Both works use biology as inspiration to discuss how fluid flows interact with the soft materials that surround them, and how these interactions give rise to non-trivial and non-monotonic changes of drag and resistance.

In the paper by Alvarado et al., the authors consider dense mats of deformable hairs subject to low-Reynolds number flows (see Fig. 1A). For their experimental setup, they use a Taylor-Couette geometry composed of two concentric cylinders rotating with respect to each other. The fluid is confined between a "hairy" surface with bendable elastomer protrusions and a smooth surface. Although the constitutive relations that govern the system are linear, the authors find that a nonlinearity in the drag reduction emerges. The vertically anchored hairs, when subject to a fluid flow, respond by bending in the direction of the flow, as shown in Fig. 1B. The deformation of the hairs increases the gap between the tip of the hairs and the smooth surface, thus decreasing the overall drag characterized by an area specific impedance $Z(v)=\frac{\tau(v)}{v}=\frac{\eta}{\Delta H(v)}$, where $\tau(v)$ is the shear stress, $v$ is the velocity of the hairy surface, $\Delta H(v)$ is the distance of the hair-tip plane to the smooth surface and $\eta$ is the fluid viscocity. Straight hairs that are cantilivered perpendicularly to the surface 
always deliver a drag reducing effect. However, hairs that are anchored at an angle to the surface as in Fig. 1C, break reflection symmetry. For moderate flow against the grain, the hairs "lift", thus decreasing $\Delta H(v)$ and increasing the drag. At higher flow rates, the hairs bend in the direction of the flow, $\Delta H(v)$ increases again and the drag reduces. By balancing the force needed to bend a hair with the force from the shear stress at the tip, and performing pertrubation analysis, the authors uncover a dimensionless velocity which controls the impedance.

The authors in Louf et al. explore a similar low Reynolds number flow interacting with a soft structure, this time arising not from bendable hairs but from perforated thin membranes. The work is motivated by the membrane pores present in many biological systems such as plasmodesmata in plants and septal nanopores in fungi, which serve to control fluid flow and prevent extreme water loss in the event of damage. In their setup, the authors consider a cylindrical elastic plate with a narrow pore in the center, a cross-section of which can be seen in Fig. 2A. A pressure is applied across the plate. At low pressure, bending dominates the deformation. If the pore is very small compared to the thickness of the membrane, the pore size decreases as in Fig. 2B, and the hydraulic resistance increases. (A discussion of bending induced aperture constriction in presented in detail in [3] for two rectangular plates separated by a narrow slit; the corners of the plates come together as the plates start deforming and the slit width decreases.) At high
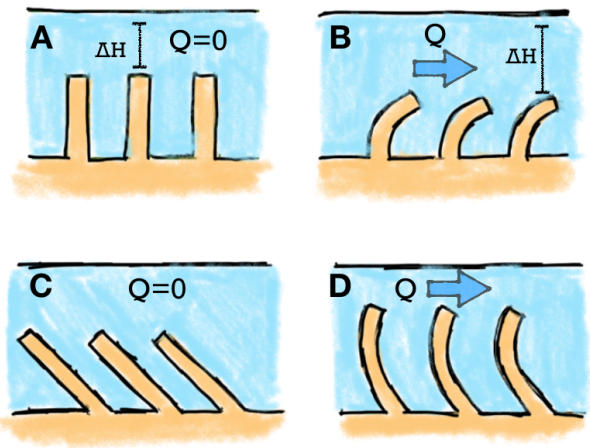

Figure 1: Schematic depicting the hair mat. (A,B) Perpendicularly anchored, zero flow at (A) and deformed filaments due to flow at (B). (C,D) Hairs anchored at an angle, zero flow at (D) and moderate flow against the grain. pressures, the membrane stretches, the pore opens, and the hydraulic resistance decreases. The authors identify the characteristic pressure that determines whether the plate will be in the stretching or the bending regime as $p_{e} \sim E d^{4} / R^{4}$, where $E$ is the Young modulus of the plate, $d$ the plate thickness, and $R$ the plate radius. Thus, the authors discover that a flexible, perforated membrane can act as a simple non-linear resistor that can passively control the flow, with fluidic resistance increasing at moderate pressures and decreasing at high pressures.

The two articles presented in this commentary are two elegant examples that demonstrate the broad phenomenology of fluid-structure interactions. They are very interesting as they point to the tantalizing observation that living organisms, being composed largely of fluids and soft elastic materials that contain them, are not only subject to these interactions, but harness them to promote a function. Alvarado et al. hypothesize that the drag reducing nonlinearity of biological hair beds such as the hyaluronan brushes of blood vessels or the brus-border microvillii of kidney tubules, protects the mechanotransducive function of these hair mats from excessive stresses. Louf et al. similarly propose that perforated plates may actually play an important role in mitigating the effects of large scale damage, and perform many fluid control functions such as delay pressure driven cytoplasmic loss. Evolution seems to be acutely aware that living tissue is largely soft and easily deformable by flows, and frequently uses simple, yet ingenuous fluid mechanical methods to achieve passive control 
devoid of biochemical complexity. By looking at how similar fluid flow problems are solved in different organisms by utilizing soft matter physics, we can learn how to design better valves and flow modulators that are cheap, effective and robust.

\section{References}

[1] M. LaBarbera, Science 249, 992 (1990).

[2] G. A. Truskey, F. Yuan, and D. F. Katz, Transport phenomena in biological systems, Pearson Prentiss Hall (2004).
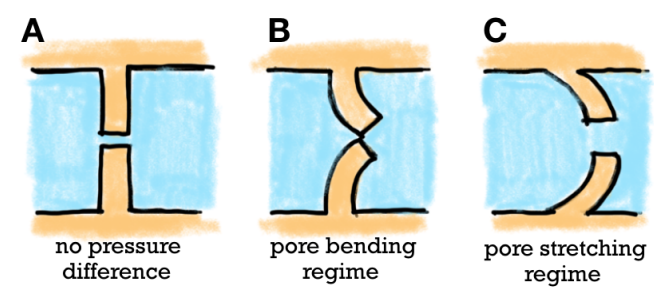

[3] A. H. Christensen and K. H. Jensen, Phys Rev Fluids, 5, 044101 (2020)

Figure 2: Schematic of the cross section of the perforated membrane inside a channel. (A) No flow. (B) Moderate pressure difference (C) High pressure difference. 